\title{
Rebuilding in the face of climate change
}

Richard J. Bell ${ }^{*, 1,4}$, Anthony Wood ${ }^{3,5}$, Jonathan Hare ${ }^{1,6,10}$, David Richardson $^{1,7}$, John Manderson ${ }^{2,8}$ and Timothy Miller ${ }^{3,9}$

${ }^{1}$ Northeast Fisheries Science Center, National Marine Fisheries Service, Narragansett, RI 02882

${ }^{2}$ Northeast Fisheries Science Center, National Marine Fisheries Service, Sandy Hook, NJ 07732

${ }^{3}$ Northeast Fisheries Science Center, National Marine Fisheries Service, Woods Hole, MA 02543

${ }^{4}$ Current address: The Nature Conservancy, Narragansett, RI 02882

${ }^{10}$ Current address: Northeast Fisheries Science Center, National Marine Fisheries Service, Woods Hole, MA 02543

5 anthony.wood@noaa.gov

6 jon.hare@noaa.gov

${ }^{7}$ david.richardson@noaa.gov

8john.manderson@noaa.gov

${ }^{9}$ timothy.j.miller@noaa.gov

November 8, 2017

${ }^{*}$ Corresponding author E-mail: rich.bell@tnc.org

Phone: 401-874-6511 


\section{Supplemental material}

\begin{tabular}{ll}
\hline IPCC Model & Model file \\
\hline $\begin{array}{l}\text { Community Climate System Model } \\
\text { Version } 4\end{array}$ & tas_Amon_CCSM4_rcp85_r1i1p1_200601-210012.nc \\
\hline $\begin{array}{l}\text { Centre National de Recherches } \\
\text { Mtorologiques }\end{array}$ & tas_Amon_CNRM-CM5_rcp85_r1i1p1_2006-209911.nc \\
\hline $\begin{array}{l}\text { Commonwealth Scientific and Indus- } \\
\text { trial Research Organization }\end{array}$ & tas_Amon_CSIR0-Mk3-6-0_rcp85_r1i1p1_2006-209911.nc \\
\hline Canadian Earth System Model & tas_Amon_CanESM2_rcp85_r1i1p1_200601-210012.nc \\
\hline $\begin{array}{l}\text { Flexible Global Ocean-Atmosphere- } \\
\text { Land System Model: Grid-point Ver- } \\
\text { sion } 2\end{array}$ & \\
\hline GFDL Coupled Physical Model & tas_Amon_GFDL-CM3_rcp85_r1i1p1_2006-209911.nc \\
\hline $\begin{array}{l}\text { NASA Goddard Institute for Space } \\
\text { Studies }\end{array}$ & tas_Amon_GISS-E2-H_rcp85_r1i1p1_2006-209911.nc \\
\hline Met Office Hadley Centre & tas_Amon_HadGEM2-CC_rcp85_r1i1p1_2006-209911.nc \\
\hline Institute Pierre Simon Laplace & tas_Amon_IPSL-CM5A-MR_rcp85_r1i1p1_2006-209911.nc \\
\hline $\begin{array}{l}\text { Model for Interdisciplinary Research } \\
\text { on Climate }\end{array}$ & tas_Amon_MIR0C5_rcp85_r1i1p1_2006-209911.nc \\
\hline Max-Planck-Institut fr Meteorologie & tas_Amon_MPI-ESM-MR_rcp85_r1i1p1_2006-209911.nc \\
\hline Meteorological Research Institute & tas_Amon_MRI-CGCM3_rcp85_r1i1p1_2006-209911.nc \\
\hline $\begin{array}{l}\text { Beijing Climate Center Climate Sys- } \\
\text { tem Model }\end{array}$ & tas_Amon_bcc-csm1-1_rcp85_r1i1p1_200601-209912.nc \\
\hline $\begin{array}{l}\text { Russian Institute for Numerical Math- } \\
\text { ematics Climate Model Version 4 }\end{array}$ & tas_Amon_inmcm4_rcp85_r1i1p1_2006-209911.nc \\
\hline
\end{tabular}

Table S1: The IPCC AR5 models used to develop the ensemble temperature projection from the Coupled Model Intercomparison Project Phase 5 (CMIP 2017). 


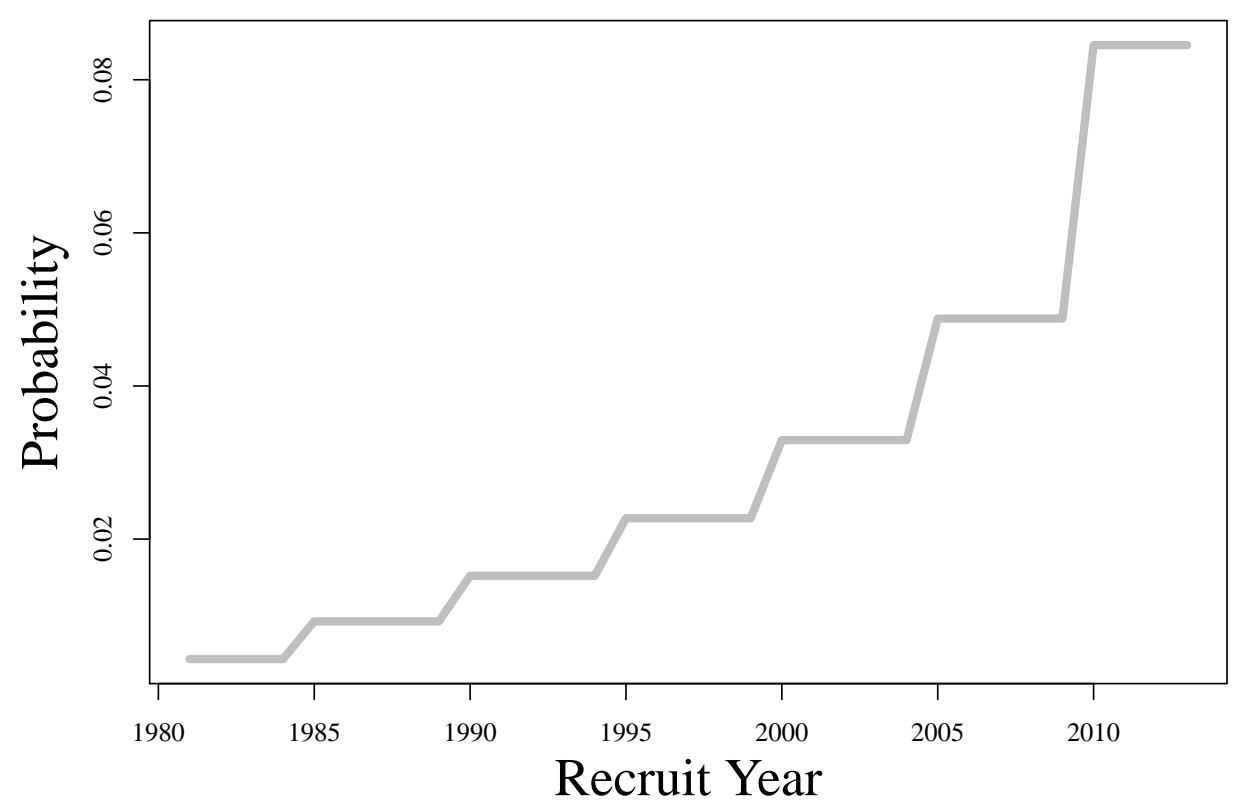

Figure S1: The probability of selecting the estimated recruitment from a given year for the ENVIRON projection. 


\section{Bootstrap runs}

Bootstrapped model runs were conducted to evaluate the difference among the estimated values (e.g. SSB, Fishing) from the three different model formulations. Because the different models were based on the same underlying data, the outputs were not independent and could not be compared with simple probability intervals. We followed the method of Miller and Legault (2017) utilizing parametric bootstrap runs. Distribution were constructed for the catch, survey indices and age composition data with the mean set to the observed values and dispersion based on the coefficient of variation. Five hundred data sets were created and run through the three different model formulations producing fifteen hundred sets of estimated values. The three different models were each fit to the same bootstrapped data set and compared. The difference in the quantities of interest (e.g. SSB, Fishing) across the different models was then sorted to produce $95 \%$ intervals.

The outputs were similar across the three models, but were consistently different after 1994 (Figures S2 \& S3). While the patterns were the same, the standard model always had the highest SSB and lowest fishing mortality while the environmental model with $\mathrm{cv}=0.2$ had the lowest SSB and highest fishing mortality. The environmental model with $\mathrm{cv}=0.5$ was in the middle. After 1994, the intervals consistently did not cross zero indicating the outputs were different among the three model formulations (Figure S4). 

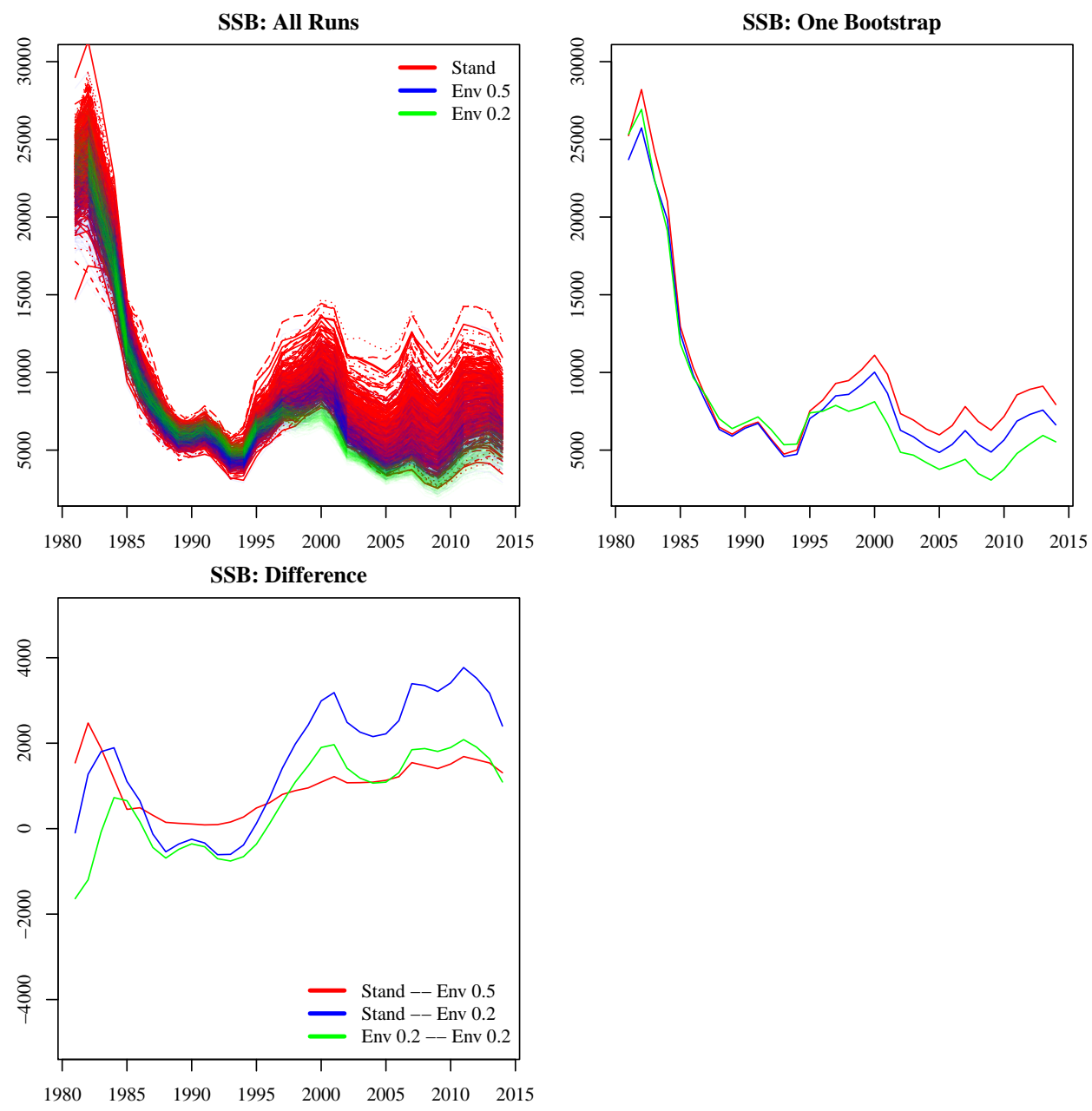

Figure S2: Estimated spawning stock biomass (SSB) for all three models across all bootstrap runs. The estimated SSB for a single bootstrap run and the difference among the estimated SSB for the particular bootstrap run. 

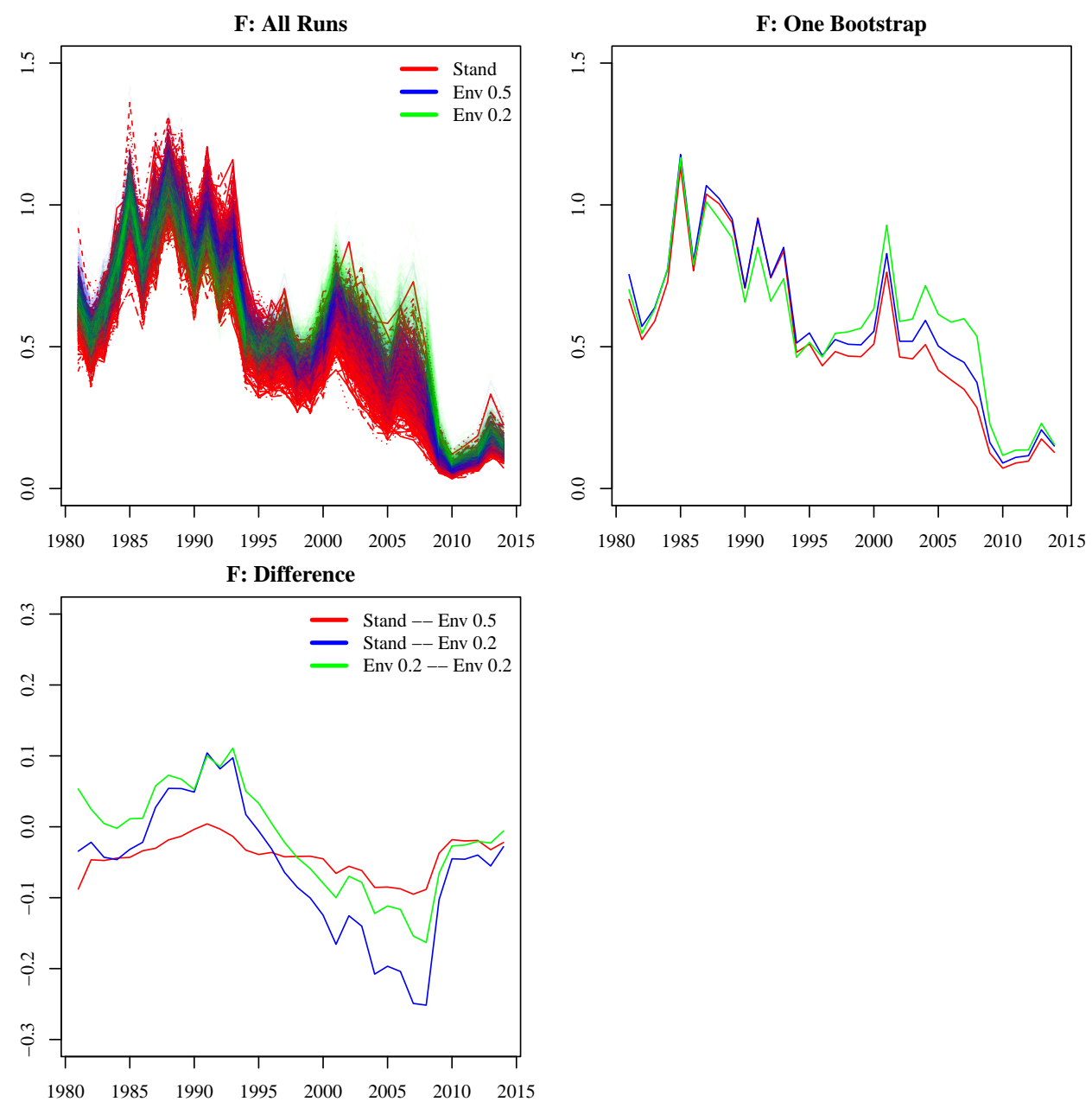

Figure S3: Estimated fishing mortality for all three models across all bootstrap runs. The estimated fishing mortality for a single bootstrap run and the difference among the estimated fishing mortality for the particular bootstrap run. 

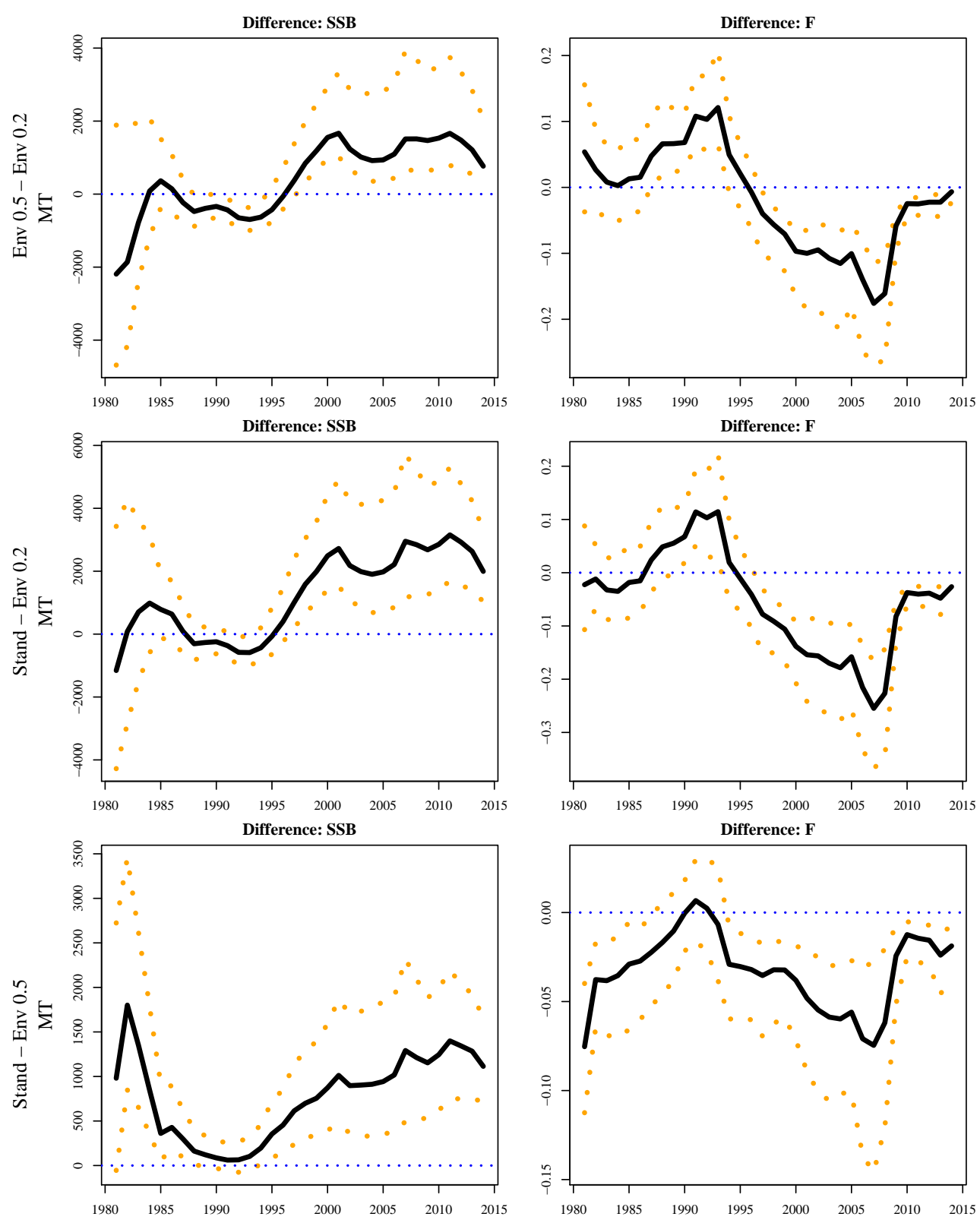

Figure S4: Estimated 95\% probability intervals and median for the difference among the three models from the parametric bootstraps. After 1994, the intervals consistently do not cross zero. 


\section{References}

CMIP 2017. http://cmip-pcmdi.llnl.gov/index.html?submenuheader $=0$.

Miller, T. and Legault, C. 2017. Statistical behavior of retrospective patterns and their effects on estimation of stock and harvest status. Fisheries Research 186(1): 109-120. 